\title{
Index
}

3D 109

3D4Medical 56

3D bioprinting 11

3D dose distributions 136

3D models 110

$3 D$ printing $4,5,63,64,65,67,69,70,71$

$3 \mathrm{D}$ virtual operating 86

anchors 92

animation 55

application 114

articulated arm 133

artificial 105

artificial intelligence 92

artificial reality 73

astigmatism 61

Atrial fibrillation 100

augmented reality (AR) 9, 10, 11, 73, 104, 154, 183

augmented visualization 111

automatic control 137

auxiliary elements 135

bedside assistance 159

biclustering 3

bioprinting 4

box trainers 157

brachytherapy 148

Brainstorm 47

calibration tools 133

cardiac interventions 96

cardiopulmonary bypass 95

C-arm 96

choreography 81

circle of learning 168

collection of waste 136

colorimetric tests 6

computer simulation 168

Conflict 77

console 86

console training 159

coronary artery bypass grafting 99

correlates of emotion 19, 27

Cox-Maze procedure 100
Crisis resource management 175

cyberknife 135

cyberspace 73

cytotoxic drugs 136

database of contractors 143

Data Matrix 139

da Vinci 119, 120, 123, 124, 125

da Vinci surgical robotic system 152

DEAP 23, 24, 25, 28

Deciding 77

decision-making $63,67,77,175,177$

decision-making process 77,78

dedicated containers 136

degrees of freedom 76

demographics data 140

Determined 77

device data 162

device malfunction 162

diagnostic 74

DICOM RT 137

Digital 105

Dipole source modeling 39

dominant rhythms 38

dorsal elevation 83

drag and drop 57

drug design 11

dual-console 161

dynamics 74

EEG lab 47

efficiency 78,91

e-learning 55, 154

electric charge flowing 41

Electroencephalography 35

emotion correlates 20, 21

empathy 170,171

endoscopic 88

endoscopic camera 131

endoscopic imaging 128

Endovascular aortic repair 100

enhanced reality 175

environment 106

ergonomics 76,78

ERP 39 
Event-related potentials 39

exercises 86

extended reality 9

extension arm 83

eyeball 57

FDA $70,71,153$

FDA-approved surgical robotic devices 151

feedback 170

FLEX 123, 125

Foundation of Cardiac Surgery Development

(Fundacja Rozwoju Kardiochirugrii im. Prof.

Zbigniewa Religi [FRK]) 75

freezing the position of instruments 132

full-body simulators 172

Fundamentals of Laparoscopic Surgery

(FLS) 150

Fundamentals of Robotic Surgery (FRS) 152

futuristic 105

General Data Protection Regulations 114

GeoSource 46

glasses 109

half-translucency 56

Heart Team 98

high-fidelity simulation 168,173

high-quality video system 133

HoloAnatomy 109

hologram 6, 92, 103, 107, 111

holographic $103,104,106,108,109,110,112$, 113, 115

holography $104,110,112,114$

HoloLens 104, 106, 108, 109, 110, 112, 113, 114, 115

home medicines 140

human eye 56

Human factor 175

hybrid 129

Hybrid coronary artery revascularization 99

hybrid models 91

hybrid operating room (OR) 9

hybrid OR 9,11

hybrid procedures 95,97

hybrid room $95,97,98,128$

hybrid specialists 98

ICA 42

image fusion 97 imaging $4,5,8,9,11,110$

imaging systems 128

immersive 107

importing/exporting data 148

Independent component analysis 42

individual processes 130

instrument arms 132

integration systems 129

intervention 90

intraoperative microscopes 128

invasiveness 90

invasive techniques 128

lowa Gambling Task 48

IT 78

keyboard 132

kinematics 74

laparoscope 79

laparoscopic 76, 132

laparoscopic skill drills 174

laparoscopy 150

learning curve 77

liver surgery 66,67

LORETA 40

manipulative 83

manual training 79

mechanical holder 133

mechanical probe 133

mechatronic $82,86,88$

medical images 108

Mentoring 161

mesh 65

mini-invasive surgical methods 79

minimal invasive surgeries 7

minimally invasive surgery 150

Mixed Reality 108

modeling $5,8,10,11,74,77$

modules 134

multidrug pills 5

muscles 57

myopia 61

nerves 57

Net Station 44

neural network 22, 27, 28

neurofeedback 29

nontechnical skills 173,183 
nurses $169,171,175$

nursing 169,171

nursing staff 141

observation of robotic surgeries 154

OpenSesame 43

operating table, like in teleoperations, introduces responsibility problems because of 78

Operation 74

optical distance 133

P300 39

palm flexion 83

pathological anatomy 57

patient simulators 169

Peyton four-step approach 181

Peyton's four-step approach 182

physical modeling 79

physical simulators 157

Picture Archiving and Communication Systems 97

planning 74

platform 86

positioning 56

power spectrum 38

precision medicine 2,6

principal component analysis 42

prognostic 74

proportions 56

psychomotor skills 158

\section{Random 77}

rare disease 3

Recognition 77

regulatory decision making 164

remote control operation 78

reoperation 73

repeatability 82

resting state 37

Robin Heart 75, 80, 81, 82, 85, 86

robot $75,76,81,82,85,88,90$

robot-assisted surgery 7,151

robotic $78,82,88$

robotic instrument 131

Robotic optics 135

Robotic surgery 151

robotic surgery registry 162

robotization 138 safety and efficacy 153

segmentation $64,69,70$

Senhance surgical robotic system 152

serious games 55

Simulated clinical conditions 168

simulated workplace 183

simulation 75,76

simulation environments 155

single-choice questions 56

single player mode 55

Situation awareness 175,176

sLORETA 40

smartphone 92

socket bone 57

Source localization 40

standardization 74,82

strategy 74

stream of information 144

students' engagement 183

surface rendering 64

surgical robots 131

surgical simulations 174

Surgical workshop 89

task trainers 170,172

TAVI 98

team leader 178

teamwork 173

technical 183

technical skill 169,180

technical skills training 180

technologies 107

technology-saturated zone 129

telemanipulation 83

telemanipulator $75,78,83,86,88$

telemedical system 86

teleoperation 77

training $77,79,80$

training and credentialing 153

trocar point 135

two-dimensional bar code 139

unfolding into parts 56

virtual 183

virtual model 55,88

virtual operating room 78

virtual reality (VR) 9, 10, 27, 73, 75, 79, 81, 82, $86,90,92,104,168$ 
virtual space $75,81,90$

visualization 8,111

visualize anatomical structures 115

visual simulation 174

VR simulator platforms 155

VR technologies 76 wearable 170

workstation 86

XR 10 